\title{
THE GEOMETRY OF THE CRITICALLY-PERIODIC CURVES IN THE SPACE OF CUBIC POLYNOMIALS
}

\author{
LAURA DE MARCO AND AARON SCHIFF
}

\begin{abstract}
We provide an algorithm for computing the Euler characteristic of the curves $\mathcal{S}_{p}$ in $\mathcal{P}_{3}^{c m} \simeq \mathbb{C}^{2}$, consisting of all polynomials with a periodic critical point of period $p$ in the space of critically-marked, complex, cubic polynomials. The curves were introduced in $\mathrm{Mi}, \mathrm{BKM}$, and the algorithm applies the main results of [DP]. The output is shown for periods $p \leq 26$.
\end{abstract}

\section{INTRODUCTION}

Let $\mathcal{P}_{3}^{c m}$ denote the space of cubic polynomials with marked critical points. It is convenient to parametrize the space $\mathcal{P}_{3}^{c m}$ by $(a, v) \in \mathbb{C}^{2}$, where the pair $(a, v)$ corresponds to the polynomial

$$
f_{a, v}(z)=z^{3}-3 a^{2} z+2 a^{3}+v
$$

with critical points at $\pm a$ and critical value $v=f_{a, v}(+a)$.

In this article, we study the geometry of the curves $\mathcal{S}_{p} \subset \mathcal{P}_{3}^{c m}$, introduced by J. Milnor in [Mi], consisting of cubic polynomials $f_{a, v}$ for which the critical point $+a$ has period exactly $p$. That is,

$$
\mathcal{S}_{p}=\left\{(a, v) \in \mathcal{P}_{d}^{c m}: f_{a, v}^{p}(a)=a, f_{a, v}^{k}(a) \neq a \text { for all } 1 \leq k<p\right\} .
$$

The curve $\mathcal{S}_{p}$ is smooth for all $p$ [Mi, Theorem 5.1]. As a (possibly disconnected) Riemann surface, the curve $\mathcal{S}_{p}$ has finite type: it is obtained from a compact Riemann surface $\overline{\mathcal{S}_{p}}$ by removing finitely many points. The punctures lie at infinity in the space $\mathcal{P}_{d}^{c m}$. To date, the irreducibility of $\mathcal{S}_{p}$ is unknown, though it is shown in [BKM, $\S 8$ ] for periods $p \leq 4$.

The goal of this article is to explain an algorithm to compute the Euler characteristic of the compactification $\overline{\mathcal{S}_{p}}$. In [BKM, Theorem 7.2], it is shown to satisfy

$$
\chi\left(\overline{\mathcal{S}_{p}}\right)=d_{p}(2-p)+N_{p} .
$$

The number $d_{p}$ is the degree of the curve $\mathcal{S}_{p}$, and it is easily computable from the defining equation. The number $N_{p}$ denotes the number of ends of $\mathcal{S}_{p}$, the punctures $\overline{\mathcal{S}_{p}} \backslash \mathcal{S}_{p}$. Our contribution is the algorithmic process to compute $N_{p}$, applying the

Date: November 7, 2018.

Research of both authors supported by the National Science Foundation. 
main results of [DP]. The Euler characteristic of $\overline{\mathcal{S}_{p}}$ is shown in Table 1, to period $p=26$.

We remark that the computation of the Euler characteristic $\chi\left(\overline{\mathcal{S}_{p}}\right)$ cannot be handled by traditional methods beyond the small periods. A quick genus computation with Maple ${ }^{\mathrm{TM}}$, for example, yielded Euler characteristics for $p \leq 4$ and failed to provide an output for $p=5$ where $\mathcal{S}_{5}$ is a curve of degree 80. The degree of $\mathcal{S}_{p}$ is on the order of $3^{p-1}$, and the curves $\overline{\mathcal{S}_{p}}$ will be highly singular at infinity for any choice of projective compactification of $\mathcal{P}_{3}^{c m} \simeq \mathbb{C}^{2}$ and $p$ sufficiently large. The Euler characteristics for periods $p \leq 4$ appear in [BKM].

1.1. Outline of the algorithm. As described in [Mi], the ends of $\mathcal{S}_{p}$ correspond to the escape regions of $\mathcal{S}_{p}$, the open subsets of $\mathcal{S}_{p}$ consisting of polynomials with the critical point $-a$ tending to infinity under iteration. The main ingredient in the computation of $N_{p}$ is the combinatorial analysis of polynomial dynamics on the basin of infinity, developed in $[\mathrm{BH}]$ and $[\mathrm{DP}]$. Recall that the basin of infinity of a polynomial $f$ is the domain

$$
X(f)=\left\{z \in \mathbb{C}: f^{n}(z) \rightarrow \infty\right\} .
$$

From $[\overline{\mathrm{BH}}]$, we use the properties of the tableau (or equivalently, the Yoccoz taufunction) of a cubic polynomial; this combinatorial object encodes the first-return of a critical point to its "critical nest." From [DP] we use the combinatorics of the pictograph, a more refined encoding of the first-return of a critical point to a "decorated critical nest," allowing us to distinguish and count topological conjugacy classes.

The steps of the algorithm are:

(1) Fix $p$. For each $k$ dividing $p$, with $1 \leq k \leq p$, determine all admissible tau-functions with period $k$.

(2) Count the number of topological conjugacy classes of basins of infinity $(f, X(f))$ associated to each tau-function.

(3) Compute the number of topological conjugacy classes of polynomials in $\mathcal{S}_{p}$ with one escaping critical point: each class is determined by the class of its basin of infinity (with a tau-function of period $k$ ) and a point in the Mandelbrot set associated to a period $p / k$ critical point.

(4) Determine the number $N_{p}$ of escape regions in $\mathcal{S}_{p}$ : there are either one or two ends in $\mathcal{S}_{p}$ associated to each topological conjugacy class computed in the previous step, determined by the twist period of the tau-function.

(5) Test the output against the degree of $\mathcal{S}_{p}: N_{p}$ is the total number of escape regions, while the degree of $\mathcal{S}_{p}$ must equal the number of escape regions counted with multiplicity. The multiplicity is computed from the tau-function.

Step (1) uses the tableau rules of [BH], as corrected in [Ki, DM]; a translation into the language of the Yoccoz tau-functions was given in [DS]. The bulk of the computing time and memory usage goes into Step (1). In $\oint_{2}$, we provide the theoretical results 


\begin{tabular}{|c|c|c|c|c|}
\hline Period & Tau-functions & Central ends & Euler characteristic & $-\chi\left(\overline{\mathcal{S}_{p}}\right) / 3^{p-1}$ \\
\hline 1 & 1 & 1 & 2 & -2.000 \\
2 & 1 & 1 & 2 & -0.667 \\
3 & 3 & 5 & 0 & 0.000 \\
4 & 6 & 13 & -28 & 1.037 \\
5 & 15 & 41 & -184 & 2.272 \\
6 & 29 & 109 & -784 & 3.226 \\
7 & 69 & 341 & -3236 & 4.439 \\
8 & 141 & 973 & -11848 & 5.417 \\
9 & 308 & 2853 & -42744 & 6.515 \\
10 & 649 & 8301 & -147948 & 7.517 \\
11 & 1406 & 24533 & -505876 & 8.560 \\
12 & 2969 & 71737 & -1694848 & 9.568 \\
13 & 6400 & 211653 & -5630092 & 10.594 \\
14 & 13636 & 623485 & -18491088 & 11.598 \\
15 & 29284 & 1842585 & -60318292 & 12.611 \\
16 & 62746 & 5447957 & -195372312 & 13.616 \\
17 & 134966 & 16134965 & -629500300 & 14.624 \\
18 & 290089 & 47820749 & -2018178784 & 15.628 \\
19 & 625298 & 141888285 & -6443997868 & 16.633 \\
20 & 1348264 & 421295297 & -20498523376 & 17.637 \\
21 & 2912779 & 1251903973 & -64995935796 & 18.641 \\
22 & 6298309 & 3722380213 & -205481381144 & 19.644 \\
23 & 13639477 & 11074683701 & -647923373764 & 20.647 \\
24 & 29567647 & 32965853477 & -2038171671252 & 21.650 \\
25 & 64181452 & 98175789309 & -6397686770076 & 22.652 \\
26 & 139464021 & 292501047833 & -20042379058084 & 23.655 \\
\hline & & & & \\
\hline
\end{tabular}

TABLE 1. The output of the Euler Characteristic algorithm. From left to right: the period $p$; the number of tau-functions with period $p$; the number of escape regions of $\mathcal{S}_{p}$ with the hybrid class of $z^{2}$ (see Theorem 5.3); the Euler characteristic $\chi\left(\overline{\mathcal{S}_{p}}\right)$; and a comparison to $3^{p-1}$.

needed for the computation. We include the theoretical results we used for improving the speed of the algorithm; we believe that some of these are interesting in their own right.

Step (2) was implemented already in [DS], applying the results of [DP]. Step (3) relies on the work of Branner and Hubbard in [BH] (see also [BKM, Theorem 3.9]), to know that the conformal class of a cubic polynomial in an escape region depends only on the class of its basin and the class of its degree 2 polynomial-like restriction. Steps (4) and (5) are explained in \$5, where we relate an escape region in $\mathcal{S}_{p}$ to its quotient 
in the moduli space of cubic polynomials $\mathcal{M}_{3}^{c m}$. The multiplicity of an escape region is computed and depends only on the underlying tau-function.

1.2. Details of the computation. An implementation of the algorithm was written with $\mathrm{C}++$. We compiled the output in Table 1 to period $p=26$. The low periods are computed quickly, while the computation for period 26 took 9:13 hours (Intel Core 2 Quad @ 2.5 GHz on Windows 7 32-bit edition), executed on a single thread.

1.3. The growth rate of $\chi\left(\overline{\mathcal{S}_{p}}\right)$. An easy computation shows that $-\chi\left(\overline{\mathcal{S}_{p}}\right) \rightarrow \infty$ as $p \rightarrow \infty$ [Mi]. Using methods from pluripotential theory, Dujardin showed that

$$
\frac{-\chi\left(\overline{\mathcal{S}_{p}}\right)}{3^{p}} \rightarrow \infty
$$

as $p \rightarrow \infty[\mathrm{Du}]$. After viewing the output of this algorithm, Milnor asked whether we have

$$
\frac{\chi\left(\overline{\mathcal{S}_{p}}\right)}{3^{p-1}}=-p+O(1)
$$

as $p \rightarrow \infty$. Or, equivalently by equation (1.1), do we have

$$
N_{p}=O\left(3^{p-1}\right) ?
$$

We include the ratio $-\chi\left(\overline{\mathcal{S}_{p}}\right) / 3^{p-1}$ in Table 1 .

1.4. Acknowledgments. We would like to thank Jan Kiwi, Jack Milnor, and Kevin Pilgrim for helpful conversations and attention to the output.

\section{The $\tau$ FUnCTIONS}

In this section, we define the Yoccoz tau-function of a cubic polynomial and explain Step 1 of the algorithm, the procedure to compute all periodic tau-functions of a given period $p$. The main theoretical result is the following:

Theorem 2.1. For each period $p \geq 1$, a tau-function has period $p$ if and only if

$$
\tau(n)=n-p
$$

for all $n \geq 2 p-2$.

We show that the bound $2 p-2$ is optimal: for every $p \geq 3$, there exists a (unique) period $p$ tau-function with $\tau(2 p-3) \neq p-3$. See Lemma 2.8 .

As described below, it is quite easy (from a theoretical point of view) to generate the periodic tau-functions, combining Theorem 2.1 with Theorem 2.2. A first approach might be to generate all admissible tau-functions of length $2 p-2$ and test for equality $\tau(2 p-2)=p-2$. As witnessed by the computations of [DS], however, the number of tau-functions grows exponentially with length, and only a small proportion are periodic. For example, there are 649 tau-functions of period $p=10$, while there are 
279,415 tau-functions of length $2 p-2=18$. Much of this section is devoted to the results we apply to reduce the computation time and memory usage.

2.1. The tau-function of a polynomial. Fix a cubic polynomial $f$ with disconnected Julia set, and let

$$
G_{f}(z)=\lim _{n \rightarrow \infty} \frac{1}{3^{n}} \log ^{+}\left|f^{n}(z)\right|
$$

be its escape rate. Let $c_{1}$ and $c_{2}$ be the critical points of $f$, labeled so that $G_{f}\left(c_{2}\right) \leq$ $G_{f}\left(c_{1}\right)$. For each integer $n \geq 0$ such that $G_{f}\left(c_{2}\right)<G_{f}\left(c_{1}\right) / 3^{n-1}$, we define the critical puzzle piece $P_{n}(f)$ as the connected component of $\left\{z: G_{f}(z)<G_{f}\left(c_{1}\right) / 3^{n-1}\right\}$ containing $c_{2}$. The puzzle piece $P_{0}(f)$ contains both critical points. For positive integers $n$, we set

$$
\tau(n)=\max \left\{j<n: f^{n-j}\left(c_{2}\right) \in P_{j}(f)\right\},
$$

defining a function $\tau$ from $\{1, \ldots, N\}$ (or all of $\mathbb{N}$ ) to the non-negative integers. The largest $N$ on which $\tau$ is defined is said to be the length of the tau-function. In other words, $N$ is the greatest integer such that $G_{f}\left(c_{2}\right)<G_{f}\left(c_{1}\right) / 3^{N-1}$. If there is no maximal $N$, we say $\tau$ has length $\infty$.

The markers of a tau-function with length $N$ are the integers

$$
\{m \in\{1, \ldots, N-1\}: \tau(m+1)<\tau(m)+1\} .
$$

The marked levels of $\tau$ are all integers in the forward orbit of a marker:

$$
\left\{l \geq 0: l=\tau^{n}(m) \text { for marker } m \text { and } n>0\right\} \cup\{0\} ;
$$

we say 0 is marked even if there are no markers. The positive marked levels coincide with the lengths of the columns in the Branner-Hubbard tableau. In terms of the polynomial $f$, a level $l$ is marked if the orbit of the critical point intersects $P_{l}(f) \backslash$ $\overline{P_{l-1}(f)}$. We say $l$ is marked by $k$ if the $k$-th iterate $f^{k}\left(c_{i}\right)$ lies in $P_{l}(f) \backslash \overline{P_{l-1}(f)}$.

2.2. Properties of tau-functions. Let $\mathbb{N}$ denote the positive integers $\{1,2,3, \ldots\}$. For any positive integer $N$, a function

$$
\tau:\{1,2,3, \ldots, N\} \rightarrow \mathbb{N} \cup\{0\}
$$

or a function

$$
\tau: \mathbb{N} \rightarrow \mathbb{N} \cup\{0\}
$$

is said to be admissible if it satisfies the following properties (A)-(E):
(A) $\tau(1)=0$
(B) $\tau(n+1) \leq \tau(n)+1$

From (A) and (B), it follows that $\tau(n)<n$ for all $n \in \mathbb{N}$; consequently, there exists a unique integer $\operatorname{ord}(n)$ such that the iterate $\tau^{\operatorname{ord}(n)}(n)=0$.

(C) If $\tau(n+1)<\tau^{k}(n)+1$ for some $0<k<\operatorname{ord}(n)$, then $\tau(n+1) \leq \tau^{k+1}(n)+1$. 
(D) If $\tau(n+1)<\tau^{k}(n)+1$ for some $0<k<\operatorname{ord}(n)$, and if $\tau\left(\tau^{k}(n)+1\right)=$ $\tau^{k+1}(n)+1$, then $\tau(n+1)<\tau^{k+1}(n)+1$.

(E) If $\operatorname{ord}(n)>1$ and $\operatorname{ord}\left(\tau^{\operatorname{ord}(n)-1}(n)+1\right)=1$, then $\tau(n+1) \neq 0$.

A tau-function is admissible if and only if it is the tau-function of a cubic polynomial [DS, Proposition 2.1]. The proof is by induction on $N$, applying the rules for admissible tableaux in $[\mathrm{BH}]$. Property (E) is another formulation of the "missing tableau rule" (M4) appearing in [Ki] and [DM].

Let $k$ be the number of markers which appear in the orbit

$$
N \mapsto \tau(N) \mapsto \ldots \mapsto \tau^{\operatorname{ord}(N)}(N)=0,
$$

and label these $k$ markers by $l_{1}^{\prime}, l_{2}^{\prime}, \ldots, l_{k}^{\prime}$ so that

$$
N=l_{0}^{\prime}>l_{1}^{\prime}>l_{2}^{\prime}>\cdots>l_{k}^{\prime}>0 .
$$

For each $0 \leq i \leq k$, let $l_{i}=\tau\left(l_{i}^{\prime}\right)$ so that

$$
\tau(N)=l_{0}>l_{1}>\cdots>l_{k} \geq 0 .
$$

Properties (A)-(E) imply the following:

Theorem 2.2. [DS, Theorem 2.2] Given an admissible tau-function $\tau$ of length $N$, an extension to length $N+1$ is admissible if and only if

$$
\tau(N+1)=l_{i}+1 \text { for some } 0 \leq i \leq k
$$

or $\tau(N+1)=0$ if $l_{k}>0$ or $k=0$.

Note, in particular, that $\tau(N+1)=\tau(N)+1$ is always an admissible extension to length $N+1$.

2.3. Periodic tau-functions. For cubic polynomials with exactly one critical point in the basin of infinity, the tau-function will have infinite length. An admissible taufunction $\tau: \mathbb{N} \rightarrow \mathbb{N} \cup\{0\}$ is periodic with period $p$ if there exists $N(\tau) \in \mathbb{N}$ such that

$$
\tau(n)=n-p
$$

for all $n \geq N(\tau)$. Such tau-functions correspond to basins of infinity with a bounded critical orbit in a periodic component of the filled Julia set; $\tau$ has period $p$ if and only if the component has period exactly $p$. For computational purposes, we need a bound on $N(\tau)$ depending only on the period $p$. The bound $N(\tau) \leq 2 p-2$ is granted by Theorem 2.1, which we prove below.

Lemma 2.3. If $\tau$ has period $p$, then $\tau(n) \geq n-p$ for all $n$. Further, if $\tau\left(n_{0}\right)=n_{0}-p$ for some $n_{0}$, then $\tau(n)=n-p$ for all $n \geq n_{0}$.

Proof. This follows easily from property (B).

Lemma 2.4. If $\tau$ has period $p$, then $l \leq p-1$ for all marked levels $l$. 
Proof. Let $f$ be any cubic polynomial with a given periodic tau-function. Label the critical points of $f$ as in $\$ 2.1$. Without loss of generality, we may assume the critical point $c_{2}$ is periodic with period exactly $p$.

Suppose $l$ is marked by iterate $k$, and assume first that $\tau(l)=0$. From Lemma 2.3. we have $l=l-\tau(l) \leq p$. The first return of $P_{l}$ to the critical nest occurs with $f^{l}\left(P_{l}\right)=P_{0}$. Because it maps with degree 2, the iterates $f^{l}\left(c_{2}\right)$ and $f^{k+l}\left(c_{2}\right)$ must lie in the two distinct components of $\left\{G_{f}<G_{f}\left(c_{1}\right)\right\}$ inside $P_{0}$. By periodicity, then, we must have $l<p$.

More generally, we have that the first return of $P_{l}$ to the critical nest is $f^{l-\tau(l)}\left(P_{l}\right)=$ $P_{\tau(l)}$, and $l-\tau(l) \leq p$. As above, because the first return is with degree 2 , the images $f^{l-\tau(l)} c_{2}$ and $f^{k+l-\tau(l)}\left(c_{2}\right)$ cannot lie in the same component of $\left\{G_{f}<G_{f}\left(c_{2}\right) / 3^{\tau(l)}\right\}$ within $P_{\tau(l)}$, while $c_{2}$ and $f^{k}\left(c_{2}\right)$ do lie in the same component. Therefore $l-\tau(l)<p$.

In addition, we must have $l-\tau^{2}(p) \leq p$, as this is the first level where the forward orbit of $c_{2}$ and $f^{k}\left(c_{2}\right)$ might come together. If $l$ is not a marker, then $f^{l-\tau(l)}\left(c_{2}\right)$ lies in the same component as $c_{2}$ at $\tau(l)$, and therefore its image at $\tau^{2}(l)$ is in a distinct component from that of $f^{k+l-\tau^{2}(l)}\left(c_{2}\right)$. On the other hand, if $l$ is a marker, then $\tau(l)$ is marked by $l-\tau(l)$. By periodicity, we can take $k=p-(l-\tau(l))$. At $\tau^{2}(l)$, we have $f^{l-\tau^{2}(l)}\left(c_{2}\right)$ and $f^{k+l-\tau^{2}(l)}\left(c_{2}\right)$ again in distinct components. In either case, we conclude that $l-\tau^{2}(l)<p$.

We continue inductively. For the induction step, we begin with $l-\tau^{n}(p)<p$ and $l-\tau^{n+1}(l) \leq p$. We observe that at level $\tau^{n-1}(l)$, either $f^{l-\tau^{n-1}(l)}\left(c_{2}\right)$ or $f^{k+l-\tau^{n-1}(l)}\left(c_{2}\right)$ lies in the same component as $c_{2}$. We consider the two cases: if $\tau^{n-1}(l)$ is not a marker, then we may proceed two iterates to $\tau^{n+1}(l)$ keeping the image components distinct. If $\tau^{n-1}(l)$ is a marker, then $\tau^{n-1}(l)$ is marked by $p-\left(\tau^{n-1}(l)-\tau^{n}(l)\right)$; the component containing $f^{p-\left(\tau^{n-1}(l)-\tau^{n}(l)\right)}\left(c_{2}\right)$ and the component containing $c_{2}$ at $\tau^{n-1}(l)$ must have distinct preimages at level $l$ which are sent to distinct components of $\tau^{n}(l)$, one of which contains $c_{2}$, and therefore to distinct components at $\tau^{n+1}(l)$. We conclude that $l-\tau^{n+1}(l)<p$.

Continuing until $\tau^{\text {ord }(l)}(l)=0$ completes the proof that $l<p$.

Lemma 2.5. If $\tau$ has period $p$, and if a level $l$ is marked by $k=p-1$, then $l \leq p-2$.

Proof. Suppose $l$ is marked by $p-1$. From Lemma 2.4, $l \leq p-1$. By periodicity, $\tau(l)=l-1$. From the admissible $\tau$ rules, it follows that $\tau(n)=n-1$ for all $1 \leq n \leq l$. It follows that $n$ cannot be a marker for any $n \leq l-1$. Consequently, level $n$ is marked by $l-n$ for all $0 \leq n \leq l-1$; in particular, $l$ marks level 0 . Therefore $l \neq p-1$, because $p-1$ marks level $l$.

Proof of Theorem 2.1. Suppose $\tau$ is periodic with period $p$. By definition, there exists $N(\tau)$ so that $\tau(n)=n-p$ for all $n \geq N(\tau)$. From Lemma 2.4, there are no marked levels $l \geq p$. Therefore, there are no markers at levels $l \geq p+p-1=2 p-1$. Consequently, $\tau(n+1)=\tau(n)+1$ for all $n \geq 2 p-1$, and so we must have $\tau(n)=n-p$ 
for all $n \geq 2 p-1$. If $2 p-2$ is a marker, then $\tau(2 p-2)=p-1$, but this would imply that level $p-1$ is marked by $p-1$, violating Lemma 2.5. Therefore, $\tau(2 p-2)=p-2$.

Lemma 2.6. Suppose $\tau$ has length $N$ and $\tau(N)=N-p$. Then $\tau$ extends uniquely to a sequence of period $p$, by setting

$$
\tau(n)=n-p
$$

for all $n>N$.

Proof. The existence of the extension follows directly from Theorem 2.2 , the uniqueness from property $(\mathrm{B})$.

Lemma 2.7. Let $\tau$ have period $p$, and suppose $\tau\left(n_{0}\right)>n_{0}-p$ and $\tau\left(n_{0}+1\right)=$ $n_{0}+1-p$. Then there exists a marker $m<p$ so that $\tau(m)=n_{0}-p$.

Proof. By periodicity, there is some iterate $k$ so that $\tau^{k}\left(n_{0}\right)=n_{0}-p$. By assumption, $k>1$. Let $m=\tau^{k-1}\left(n_{0}\right)$. Because $\tau\left(n_{0}+1\right)=n_{0}-p+1$, we have that $n_{0}$ is a marker, so $m$ is marked. By Lemma 2.4, then, $m<p$. We need to show $m$ is also a marker. Indeed, $\tau(m+1)=\tau\left(\tau^{k-1}\left(n_{0}\right)+1\right) \neq n_{0}-p+1$ by property $(\mathrm{D})$.

2.4. Examples/Exceptions. As demonstrated in Theorem 2.1, all periodic taufunctions of period $p$ must satisfy $\tau(n)=n-p$ for all $n \geq 2 p-2$. In fact, most periodic tau-functions of period $p$ also satisfy $\tau(n)=n-p$ for all $n \geq 2 p-5$. The following lemmas provide a complete list of the exceptions. In the lemmas, we express the tau-function as a sequence of the form $\tau(1), \tau(2), \tau(3), \cdots$. We remark that these lemmas are not used in the algorithm for the Euler characteristic computation, but we include them for completeness.

Lemma 2.8. For each period $p \geq 3$, there is a unique periodic tau-function with $\tau(n)=n-p$ for all $n \geq 2 p-2$ and $\tau(2 p-3) \neq p-3$. It is given by

- $0,1,2, \cdots, p-3,0,1,2, \cdots, p-2, p-2, p-1, p, \cdots$.

Proof. By Lemma 2.7, there is a marker $m<p$ with $\tau(m)=p-3$. Thus $m$ can only be $p-2$ or $p-1$. Consequently, the tau-function must begin with $0,1,2, \ldots,(p-3)$ or with $0,0,1, \ldots,(p-3)$. In the first case, Theorem 2.2 implies that it can only be extended as $0,1,2, \ldots,(p-3), 0$ with $\tau(2 p-3)=p-2$ and $\tau(2 p-2)=p-2$. In the case of $0,0,1,2, \ldots,(p-3)$, if $p$ is even, then Theorem 2.2 implies the extension must be as $0,0,1, \ldots,(p-3), 1,2, \ldots$, with $\tau(2 p-3)=p-2$, but we cannot extend by $\tau(2 p-2)=p-2$. If $p$ is odd, then we must have $\tau(p)=0$, but then $\tau(n)=n-p$ for all $n \geq p$.

Lemma 2.9. For each period $p \geq 4$, the only periodic tau-functions with $\tau(n)=n-p$ for all $n \geq 2 p-3$ and $\tau(2 p-4) \neq p-4$ are

- $0,1,2, \cdots, p-4,0,0,1,2, \cdots, p-3, p-3, p-2, p-1, \cdots$;

- $0,1,2, \cdots, p-4,0,1,2, \cdots, p-3, p-3, p-3, p-2, p-1, \cdots$ 
and if $p$ is odd then also

- $0,0,1,2, \cdots, p-4,1,2, \cdots, p-2, p-3, p-2, p-1, \cdots$.

Proof. By Lemma 2.4, we must have $\tau(2 p-4)=p-3$ or $p-2$ or $p-1$. Also, by Lemma 2.7, level $p-4$ is marked by a marker $m<p$.

Assume $\tau(2 p-4)=p-3$. Then $p-3$ is marked by $p-1$. Periodicity implies that $p-4$ is marked by 1 ; that is $\tau(p-3)=p-4$. The $\tau$ rules then imply that $\tau(n)=n-1$ for all $1 \leq n \leq p-3$, so our tau-function begins as $0,1,2, \ldots,(p-4)$. Because $p-4$ must be marked, Theorem 2.2 implies that $\tau(p-2)=0$. Theorem 2.2 then allows for $\tau(p-1)=0$ or 1 . In either case, the tau-function is then uniquely determined by Theorem 2.2 and Lemma 2.3 , giving the first two possibilities stated in the Lemma.

Now assume $\tau(2 p-4)=p-2$. Then level $p-2$ is marked by $p-2$, so by periodicity, we must have $\tau(p-2)=p-3$ or $\tau(p-2)=p-4$. If $\tau(p-2)=p-3$, then the tau-function begins with $0,1,2, \ldots, p-3$, but then $p-4$ cannot be marked by a marker $m<p$ (contradicting Lemma 2.7). We must have $\tau(p-2)=p-4$, and the tau-function begins as $0,0,1,2, \ldots, p-4$. If $p$ is even, then we can only extend by 0 (for $p-4$ to be marked), but then $\tau(2 p-4) \leq p-3$. If $p$ is odd, then we can extend by $\tau(p-1)=1$, and the final tau-function stated in the Lemma is admissible.

The final possibility is that $\tau(2 p-4)=p-1$. The only way to mark $p-4$ by a marker $m<p$ is for $\tau(p-1)=p-4$, so the $\tau$ sequence begins with $0, \tau_{2}, \tau_{3}, 1,2, \ldots, p-4$, for some $\tau_{2}, \tau_{3} \leq 1$. Then, as $p-4$ is marked by $p-1$, we must have $\tau(p) \leq 2$ by Theorem 2.2. But then $\tau(2 p-4)<p-1$, so its $\tau$ orbit does not encounter any markers larger than 1 , and we cannot have $\tau(2 p-3)=p-3$.

Lemma 2.10. For each period $p \geq 5$, the only periodic tau-functions with $\tau(n)=n-p$ for all $n \geq 2 p-4$ and $\tau(2 p-5) \neq p-5$ are

- $0,1,2, \cdots, p-5,0,1,2, \cdots, p-4, p-4, p-4, p-3, p-2, \cdots$;

- $0,1,2, \cdots, p-5,0,0,1,2, \cdots, p-4, p-4, p-4, p-3, p-2, \cdots$;

- $0,1,2, \cdots, p-5,0,0,0,1,2, \cdots, p-4, p-4, p-3, p-2, \cdots$;

- $0,1,2, \cdots, p-5,0,1,0,1,2, \cdots, p-4, p-4, p-3, p-2, \cdots$;

and if $p$ is odd, then also

- $0,0,1,2, \cdots, p-5,0,1,2, \cdots, p-3, p-4, p-3, p-2, \cdots$;

and if $p$ is even, then also

- $0,0,1,2, \cdots, p-5,1,1,2, \cdots, p-3, p-4, p-3, p-2, \cdots$;

and if $(p-1)$ is divisible by 3 , then also

- $0,1,0,1,2, \cdots, p-5,2,3, \cdots, p-2, p-4, p-3, p-2, \cdots$;

and if $(p-2)$ is divisible by 3 , then also

- $0,0,1,1,2, \cdots, p-5,2,3, \cdots, p-2, p-4, p-3, p-2, \cdots$. 
Proof. By Lemma 2.7, $p-5$ is marked by a marker $<p$. By Lemma 2.4, we must have $\tau(2 p-5)$ equal to $p-4, p-3, p-2$, or $p-1$.

Assume $\tau(2 p-5)=p-4$. Then level $p-4$ is marked by $p-1$. Periodicity implies that $\tau(p-4)=p-3$, and therefore that $\tau(n)=n-1$ for all $n \leq p-4$. Therefore, $\tau$ begins with $0,1,2, \ldots, p-5,0$. To reach $\tau(2 p-5)=p-4$, we must have $1 \leq \tau(p) \leq 3$, allowing only the first four possibilities listed in the Lemma.

Assume $\tau(2 p-5)=p-3$. We must have $\tau(p-3)$ equal to $p-4$ or $p-5$, by periodicity; for $p-5$ to be marked, we must have $\tau(p-3)=p-5$. The tau-function begins with $0,0,1,2, \ldots, p-5$. If $p$ is odd, it can be continued by setting $\tau(p-2)=0$, $\ldots, \tau(2 p-5)=p-3$, and $\tau(2 p-4)=p-4$. If $p$ is even, then $\tau(p-2)=1$, so we can take $\tau(p-1)=1$ to allow for $\tau(2 p-5)=p-3$.

A similar argument handles the case of $\tau(2 p-5)=p-2$.

\section{Generating Periodic tau-Functions}

The goal is to generate a list of all tau-functions of period $p$. For the later steps in the algorithm, we need the data of the tau-functions themselves, not only the total number.

There is a unique tau-function of period $p=1$, given by $\tau(n)=n-1$ for all $n \geq 1$. For small periods, say period $p \leq 10$, there are few periodic tau-functions. Applying Theorem 2.2, we can generate all tau-functions to length $2 p-2$. From Theorem 2.1, the equality $\tau(2 p-2)=p-2$ holds if and only if this tau-function extends to a sequence of period $p$; further, the extension is uniquely determined. For example, the total number of tau-functions of length $8(=2 p-2$ for $p=5)$ is only 144 , so the computation time and memory usage are negligible for period $p=5$ [DS]. As the period grows, the total number of tau-functions of length $2 p-2$ grows fast; it is probably larger than $4^{p-1}$. We use the Lemmas of the previous section to reduce our computational requirements.

3.1. Algorithm. The algorithm proceeds as follows. Fix $p>1$. We generate a list called Periodic containing all tau-functions of period $p$. For the induction step, we generate a list called Continue.

Initialization. Generate all tau-functions to length $n=p$, following Theorem 2.2. If $\tau(p)=0$, include in Periodic. If $\tau$ has no markers, then discard. Otherwise, include in Continue.

Extension to length $n+1$ and test for periodicity. Choose $\tau$ from the list Continue. Let $n$ be its length; by construction, $\tau(n)>$ $n-p$. Determine values $l_{0}, l_{1}, \ldots$ (as appearing in Theorem 2.2 , setting 
$N=n$ ) subject to the extra condition $n-p \leq l_{i} \leq l_{0}=\tau(n)$. For each such $l_{i}$, we consider the admissible extension of $\tau$, defined by

$$
\tau(n+1)=l_{i}+1 \text {. }
$$

If $l_{i}=n-p$, then include the extended $\tau$ in Periodic; by Lemma 2.6. this $\tau$ uniquely determines a periodic tau-function.

If $n<2 p-3$ and if $l_{i}>n-p$ and if

$$
\max \{\tau(m): m<p, \tau(m+1) \leq \tau(m)\}>n-p,
$$

then include in Continue; this $\tau$ is a candidate to have a periodic extension, as it satisfies the necessary conditions of Lemmas 2.3 and 2.7 and Theorem 2.1. Otherwise, discard. Repeat the induction step until Continue is empty.

3.2. Details. In Tables 2 and 3, we include the particulars of our computation for generating all periodic tau-functions of periods 10 and 20. Following the algorithm above, we show the number of tau-functions in the lists Periodic and Continue as we increase the length of the tau-functions.

\begin{tabular}{|c|c|c|c|}
\hline Length & Periodic & Discard & Continue \\
\hline 10 & 205 & 1 & 435 \\
11 & 201 & 242 & 506 \\
12 & 139 & 567 & 479 \\
13 & 57 & 780 & 279 \\
14 & 26 & 497 & 134 \\
15 & 12 & 251 & 61 \\
16 & 6 & 122 & 21 \\
17 & 2 & 43 & 6 \\
18 & 1 & 13 & 0 \\
\hline
\end{tabular}

TABLE 2. Period 10 details: generating the 649 tau-functions of period 10 from a total of 279,415 tau-functions of length 18. Final data file size $=7.8 \mathrm{~KB}$, peak disk usage $=18 \mathrm{~KB}$.

\section{TOPOlOgicAl CONJUGACY ClASSES OF BASINS}

In this section, we describe the algorithm to compute the number $\operatorname{Top}(\tau)$ of topological conjugacy classes of basins $(f, X(f))$ with a given tau-function $\tau$. It is proved in $[\mathrm{DP}]$ that $\operatorname{Top}(\tau)$ can be computed as

$$
\operatorname{Top}(\tau)=\operatorname{Spines}(\tau) \cdot \operatorname{TF}(\tau)
$$




\begin{tabular}{|c|c|c|c|}
\hline Length & Periodic & Discard & Continue \\
\hline 20 & 449308 & 1 & 848362 \\
21 & 319756 & 528624 & 1055320 \\
22 & 389254 & 1059653 & 1116657 \\
23 & 114128 & 1523035 & 978211 \\
24 & 41925 & 1646071 & 674730 \\
25 & 17081 & 1299907 & 391444 \\
26 & 8896 & 800601 & 196937 \\
27 & 4138 & 403194 & 93346 \\
28 & 1898 & 192799 & 44601 \\
29 & 978 & 92478 & 20839 \\
30 & 475 & 43078 & 9636 \\
31 & 217 & 20028 & 4571 \\
32 & 113 & 9623 & 2054 \\
33 & 52 & 4309 & 932 \\
34 & 24 & 2004 & 414 \\
35 & 12 & 901 & 169 \\
36 & 6 & 373 & 57 \\
37 & 2 & 137 & 18 \\
38 & 1 & 41 & 0 \\
\hline
\end{tabular}

TABLE 3. Period 20 details: generating the 1,348,264 tau-functions of period $p=20$ from a total of about 1.5 trillion tau-functions of length $2 p-2=38$. Final data file size $=29 \mathrm{MB}$, peak disk usage $=74 \mathrm{MB}$.

where $\operatorname{Spines}(\tau)$ is the number of pictographs (or truncated spines) associated to $\tau$ and $\mathrm{TF}(\tau)$ is the associated twist factor. We include here the steps to compute $\operatorname{Spines}(\tau)$ and $\operatorname{TF}(\tau)$. These details already appeared in [DS].

The twist factor $\operatorname{TF}(\tau)$ is denoted by $\operatorname{Top}(\mathcal{D})$ in $[\mathrm{DP}]$, the number of conjugacy classes of basins with pictograph $\mathcal{D}$, for any pictograph with tau-function $\tau$. Indeed, it is easy to see that any pictograph with a period tau-function will have only finitely many marked levels, thus satisfying the hypotheses of [DP, Theorem 9.1]; further, it is stated there that the computation of $\operatorname{Top}(\mathcal{D})$ depends only on the underlying tau-function.

4.1. Computing the number of pictographs. Fix an admissible tau-function $\tau$ of length $N$. As in $\$ 2.1$, the markers of $\tau$ are the integers

$$
\{m \in\{1, \ldots, N-1\}: \tau(m+1)<\tau(m)+1\} .
$$

The marked levels of $\tau$ are all integers in the forward orbits of the markers:

$$
\left\{l \geq 0: l=\tau^{n}(m) \text { for marker } m \text { and } n>0\right\} \cup\{0\} ;
$$


we say 0 is marked even if there are no markers.

As in Theorem 2.2, we let $k$ be the number of markers which appear in the orbit

$$
N \mapsto \tau(N) \mapsto \ldots \mapsto \tau^{\operatorname{ord}(N)}(N)=0 .
$$

Label these $k$ markers by $l_{1}^{\prime}, l_{2}^{\prime}, \ldots, l_{k}^{\prime}$ so that

$$
N=l_{0}^{\prime}>l_{1}^{\prime}>l_{2}^{\prime}>\cdots>l_{k}^{\prime}>0
$$

For each $0 \leq i \leq k$, let $l_{i}=\tau\left(l_{i}^{\prime}\right)$ so that

$$
\tau(N)=l_{0}>l_{1}>\cdots>l_{k} \geq 0 .
$$

For each $0 \leq i<k$, define $n_{i}$ by the condition that

$$
\tau^{n_{i}}\left(l_{i}\right)=l_{i+1}
$$

and define $n_{k}$ so that $\tau^{n_{k}}\left(l_{k}\right)=0$. (The $n_{i}$ are called special orders in the program.) For $0<i<j \leq k+1$, we set

$$
\delta(i, j)= \begin{cases}1 & \text { if } \tau\left(l_{i}^{\prime}+1\right)=l_{j}+1 \\ 0 & \text { otherwise }\end{cases}
$$

where by convention we take $l_{k+1}=-1$. Note that $\tau\left(l_{k}^{\prime}+1\right)=0$ for every $\tau$, so $\delta(k, k+1)=1$.

The symmetry of $\tau$ is

$$
s=\min \left\{n \geq 0: \tau^{n}\left(l_{0}\right) \text { is a marked level }\right\} .
$$

Note that $s \leq n_{0}$. To each admissible choice for $\tau(N+1)$ (from Theorem 2.2) we define the $(N+1)$-th spine factor of $\tau$. If $\tau(N+1)=l_{i}+1$ with $i>0$, we set

$$
\mathrm{SF}(\tau, N+1):=2^{n_{0}-s}\left(2^{n_{1}}\left(2^{n_{2}}\left(\cdots\left(2^{n_{i-1}}-\delta(i-1, i)\right)-\cdots\right)-\delta(2, i)\right)-\delta(1, i)\right) ;
$$

as above, we take $l_{k+1}=-1$. If $\tau(N+1)=l_{0}+1=\tau(N)+1$, we set

$$
\mathrm{SF}(\tau, N+1)=1
$$

The number of pictographs (or equivalently, truncated spines) associated to a taufunction is computed inductively on the length.

Proposition 4.1. Let $\tau$ be a periodic tau-function of period $p$. The number of pictographs with tau-function $\tau$ is given by

$$
\operatorname{Spines}(\tau)=\prod_{j=1}^{N} \mathrm{SF}(\tau, j)
$$

for any choice of $N$ with $\tau(N)=N-p$. 
Proof. That $\operatorname{Spines}(\tau)$ is the product of spine factors is deduced in [DS]. It remains to show that the computation terminates at a finite $N$ when $\tau$ is periodic. From the definition of the spine factor, it is equal to 1 whenever $\tau(N+1)=\tau(N)+1$. For periodic taus, this will be the case for all $N$ sufficiently large. Recall from Lemma 2.3 that once we find one $N$ with $\tau(N)=N-p$, this equality will hold for all $n \geq N$.

4.2. Computing the twist factor. Fix an admissible tau-function $\tau$ of length $N \in$ $\mathbb{N} \cup\{\infty\}$ with finitely many marked levels. For each $n<N$, the order of $n$ was defined in 92.2 ; it satisfies $\tau^{\text {ord }(n)}(n)=0$. For each marked level $l>0$, compute

$$
\bmod (l)=\sum_{i=1}^{l} 2^{-\operatorname{ord}(i)}
$$

and

$$
t(l)=\min \{n>0: n \bmod (l) \in \mathbb{N}\} .
$$

We define the twist period $T(\tau)$ by

$$
T(\tau)=\max \{t(l): l \text { is a marked level }\}
$$

or set $T(\tau)=1$ if $\tau$ has no non-zero marked levels.

Let $L(\tau)$ be the number of non-zero marked levels. The twist factor is defined by

$$
\mathrm{TF}(\tau)=\frac{2^{L(\tau)}}{T(\tau)} .
$$

Theorem 9.1 of [DP] states that the number of topological conjugacy classes of basins associated to a given pictograph with tau-function $\tau$ is equal to $\operatorname{TF}(\tau)$.

4.3. The significance of the twist factor. We include a few words here to explain the meaning of the values appearing in $\$ 4.2$ to define the twist factor. These play a role in the explanations of $\$ 5$.

The quasiconformal deformations of the basin of infinity of a polynomial $f$ have a natural decomposition into twisting and stretching factors; see [McS] or the summary in $[\mathrm{DP}]$. Let $f=f_{(a, v)}$ be a cubic polynomial with periodic tau-function $\tau$. Let $G_{f}$ be its escape-rate function. Recall that $-a$ is the critical point that escapes to infinity. The fundamental annulus of $f$ is the domain

$$
A(f)=\left\{z \in \mathbb{C}: G_{f}(-a)<G_{f}(z)<3 G_{f}(-a)\right\} .
$$

Viewing the basin of infinity $X(f)$ as an abstract Riemann surface, a full Dehn twist in $A(f)$ induces the hemidromy action described in $[\mathrm{BH}]$; see also $[\mathrm{Br}]$ for an accessible summary.

The twist period $T(\tau)$ is the least power of a full Dehn twist in the fundamental annulus that lies in the mapping class group of $f$. To compute $T(\tau)$, we determine the induced amount of twisting in any image or preimage of $A(f)$ under the action of $f$. Following the descriptions in $[\mathrm{Br}]$ and $[\mathrm{BH}]$, it suffices to compute the relative 
moduli of these annuli lying between the two critical points; the relative modulus of an annulus $A$ is the ratio $\bmod (A) / \bmod (A(f))$. The value $\bmod (l)$ computes exactly these sums of relative moduli down to the $l$-th marked level.

The twist factor is the ingredient emphasized in [DP. By measuring twist periods against the total number of ways to produce basins $(f, X(f))$ from a given pictograph, the discrepancy amounts to the twist factor $\mathrm{TF}(\tau)$.

\section{ESCAPE REGIONS}

In this section we explain the final steps of the algorithm, incorporating the computations described in the previous section.

5.1. The moduli space. As discussed in [Mi], there is a natural involution on the space $\mathcal{P}_{3}^{c m}$, given by

$$
I(a, v)=(-a,-v)
$$

induced by the conjugation of $f_{(a, v)}$ by $z \mapsto-z$. Thus there is a degree 2 projection

$$
\mathcal{P}_{3}^{c m} \rightarrow \mathcal{P}_{3}^{c m} / I=: \mathcal{M}_{3}^{c m}
$$

to the moduli space of critically-marked cubic polynomials. The action of $I$ preserves the curve $\mathcal{S}_{p}$, defining a curve $\mathcal{S}_{p} / I \subset \mathcal{M}_{3}^{c m}$.

5.2. Escape regions and multiplicity. As introduced in [Mi] and [BKM], an escape region of $\mathcal{S}_{p}$ is a connected component of

$$
\left\{f_{(a, v)} \in \mathcal{S}_{p}: f_{(a, v)}^{n}(-a) \rightarrow \infty \text { as } n \rightarrow \infty\right\} .
$$

That is, it consists of maps with one periodic critical point $($ at $+a)$ and one escaping critical point (at $-a)$.

It follows from the general theory on stability that all polynomials in a given escape region $E$ are topologically conjugate on $\mathbb{C}$, as described in [McS]. In this special setting, though, it can be seen directly from a canonical parameterization of $E$. It is shown in [Mi, Lemma 5.6] that each escape region $E$ is conformally a punctured disk, canonically identified with an $m$-fold cover of a punctured disk, for some positive integer $m=m(E)$. This number $m(E)$ is called the multiplicity of $E$.

The covering map of degree $m(E)$ is defined by the assignment

$$
(a, v) \mapsto \varphi_{(a, v)}(2 a),
$$

where $\varphi_{(a, v)}$ defines the uniformizing Böttcher coordinates near infinity for $f_{(a, v)}$, where $\varphi_{(a, v)}\left(f_{(a, v)}(z)\right)=\left(\varphi_{(a, v)}(z)\right)^{3}$, unique if chosen to satisfy $\varphi^{\prime}(\infty)=1$. The point $2 a$ is the cocritical point for $-a$, so $f_{(a, v)}(2 a)=f_{(a, v)}(-a)$. In particular, the twisting deformation on the basin of infinity induces the change in angular coordinate on $E$. In fact, the external angle of $2 a$ is increased by $\pi$ under a full Dehn twist in the fundamental annulus of $f_{(a, v)}$; thus, $2 m(E)$ full twists closes a loop in $E$. 
Lemma 5.1. Fix an escape region $E$ and let $\tau$ be the tau-function of any $f \in E$. The multiplicity is given by

$$
m(E)= \begin{cases}1 & \text { if } T(\tau)=1 \\ T(\tau) / 2 & \text { if } T(\tau)>1\end{cases}
$$

where $T(\tau)$ is the twist period computed in \$4.2.

Proof. Each escape region $E$ projects to an escape region $E / I$ in the curve $\mathcal{S}_{p} / I \subset$ $\mathcal{M}_{3}^{c m}$. By definition of the twist period, $T(\tau)$ full twists in a fundamental annulus are required to induce a closed loop in $E / I$. But $E / I$ is doubly covered by a single escape region $E$ if and only if $f_{(a, v)}$ and $f_{(-a,-v)}$ are equivalent under a twist deformation, if and only if we have $T(\tau)=1$. In this case of $T(\tau)=1$, two full twists are required to close a loop in $E$, corresponding to an argument increase of $2 \pi$ for the cocritical point $2 a$. Therefore $m(E)=1$. On the other hand, if $T(\tau)>1$, then each escape region $E$ projects bijectively to $E / I$; thus $2 m(E)=T(\tau)$.

5.3. Hybrid classes. For any polynomial $f$ in an escape region $E$ in $\mathcal{S}_{p}$, the associated tau-function will have period $k$ for some $k$ dividing $p$. A restriction of the iterate $f^{k}$ to a certain neighborhood of $+a$ will then define a quadratic polynomial-like map. We refer to [DH] for background information. In this context, it is important to know that the conformal conjugacy class of $f$ is uniquely determined by the conformal conjugacy class of its basin of infinity $(f, X(f))$ and the hybrid class of its polynomial-like restriction [BH]. See also [BKM, Theorem 3.9, Corollary 3.10].

We will use the following consequence of the general theory:

Proposition 5.2. An escape region $E / I$ in $\mathcal{S}_{p} / I$ is uniquely determined by

(1) an integer $k$ dividing $p$ with $1 \leq k \leq p$;

(2) a topological conjugacy class of basin dynamics $(f, X(f))$ with a critical end of period $k$; and

(3) a point in the Mandelbrot set corresponding to a center of period exactly $p / k$.

A center of period $n$ in the Mandelbrot set is a solution $c$ to the equation $f_{c}^{n}(0)=0$ where $f_{c}(z)=z^{2}+c$. The center $c$ has period exactly $n$ if $n$ is the smallest positive integer for which the equality $f_{c}^{n}(0)=0$ holds. The number $\nu_{2}(n)$ of centers of period exactly $n$ is easily computable by the following relation:

$$
2^{n-1}=\sum_{q \mid n, 1 \leq q \leq n} \nu_{2}(q)
$$

Combining the above results, we deduce the following:

Theorem 5.3. For any tau-function $\tau$ with period $k$ dividing $p$, the number of escape regions in $\mathcal{S}_{p}$ with tau-function $\tau$ is

$$
\operatorname{Ends}(\tau, p)= \begin{cases}\nu_{2}(p / k) \operatorname{Spines}(\tau) \mathrm{TF}(\tau) & \text { if } T(\tau)=1 \\ 2 \nu_{2}(p / k) \operatorname{Spines}(\tau) \mathrm{TF}(\tau) & \text { if } T(\tau)>1\end{cases}
$$


where $T(\tau)$ is the twist period, Spines $(\tau)$ is the number of pictographs, and $\mathrm{TF}(\tau)$ is the twist factor of $\tau$. The total number of escape regions in $\mathcal{S}_{p}$ is therefore

$$
N_{p}=\sum_{k \mid p} \sum_{\operatorname{per}(\tau)=k} \operatorname{Ends}(\tau, p)
$$

In particular, in the case of $k=p, \operatorname{Ends}(\tau, p)$ is the number of "central ends" of $\tau$, coinciding with the number of all escape regions of $\mathcal{S}_{p}$ with tau-function $\tau$ and hybrid class $z^{2}$. The sum of $\operatorname{Ends}(\tau, p)$ over all taus with period $p$ is shown in Table 1. The sum of $\operatorname{Ends}(\tau, p)$ over all taus with period dividing $p$ is the total number $N_{p}$ of escape regions in $\mathcal{S}_{p}$.

Proof. Fix $\tau$ of period $k$ dividing $p$. From the $\operatorname{arguments}$ of $\$ 4$, there are $\operatorname{Spines}(\tau) \operatorname{TF}(\tau)$ topological conjugacy classes of basins $(f, X(f))$ of cubic polynomials with taufunction $\tau$. Applying Proposition 5.2 , there are consequently $\nu_{2}(p / k) \operatorname{Spines}(\tau) \operatorname{TF}(\tau)$ escape regions $E / I$ in $\mathcal{S}_{p} / I \subset \mathcal{M}_{3}^{c m}$. If $T(\tau)=1$, then exactly as in the proof of Lemma 5.1, there is a unique escape region $E$ in $\mathcal{S}_{p}$ mapped to each $E / I$. If $T(\tau)>1$, there are exactly two escape regions mapped to each $E / I$.

5.4. Testing the computation. We conclude with an explanation of the test of our computation against the degree of $\mathcal{S}_{p}$.

The multiplicity of an escape region $E$ in $\mathcal{S}_{p}$ coincides with the number of intersection points of $E$ with any line in $\mathcal{P}_{3}^{c m}$ of the form $\left\{a=a_{0}\right\}$ for any $a_{0}$ of sufficiently large modulus. Therefore, the degree $d_{p}$ of the curve $\mathcal{S}_{p}$ must satisfy

$$
d_{p}=\sum_{E} m(E)
$$

summing over all escape regions $E$ of $\mathcal{S}_{p}$. The degree $d_{p}$ is easily computed, as it satisfies:

$$
3^{p-1}=\sum_{q \mid p} d_{q}
$$

where the sum is taken over all $q$ dividing $p$ with $1 \leq q \leq p$. As established by Lemma 5.1, the value $m(E)$ depends only on the tau-function for the escape region $E$, so we may define

$$
m(\tau):=m(E)
$$

for any escape region $E$ associated to tau-function $\tau$.

Our algorithm determines the value $\operatorname{Ends}(\tau, p)$ for every tau-function of period $k$ dividing $p$; the ingredients are listed in Theorem 5.3. We can therefore check our computation by assuring equality of

$$
\sum_{\tau} m(\tau) \operatorname{Ends}(\tau, p)=d_{p}
$$

summing over all tau-functions $\tau$ of periods dividing $p$. 


\section{REFERENCES}

[BKM] A. Bonifant, J. Kiwi, and J. Milnor. Cubic polynomial maps with periodic critical orbit. II. Escape regions. Conform. Geom. Dyn. 14(2010), 68-112.

[Br] B. Branner. Cubic polynomials: turning around the connectedness locus. In Topological methods in modern mathematics (Stony Brook, NY, 1991), pages 391-427. Publish or Perish, Houston, TX, 1993.

[BH] B. Branner and J. H. Hubbard. The iteration of cubic polynomials. II. Patterns and parapatterns. Acta Math. 169(1992), 229-325.

[DM] L. DeMarco and C. McMullen. Trees and the dynamics of polynomials. Ann. Sci. École Norm. Sup. 41(2008), 337-383.

[DP] L. DeMarco and K. Pilgrim. The classification of polynomial basins of infinity. Preprint, 2011.

[DS] L. DeMarco and A. Schiff. Enumerating the basins of infinity of cubic polynomials. J. Difference Equ. Appl. 16(2010), 451-461.

[DH] A. Douady and J. H. Hubbard. On the dynamics of polynomial-like mappings. Ann. Sci. École Norm. Sup. (4) 18(1985), 287-343.

[Du] R. Dujardin. Cubic polynomials: a measurable view of parameter space. In Complex dynamics, pages 451-489. A K Peters, Wellesley, MA, 2009.

[Ki] J. Kiwi. Puiseux series polynomial dynamcs and iteration of complex cubic polynomials. Ann. Inst. Fourier (Grenoble) 56(2006), 1337-1404.

[McS] C. T. McMullen and D. P. Sullivan. Quasiconformal homeomorphisms and dynamics. III. The Teichmüller space of a holomorphic dynamical system. Adv. Math. 135(1998), 351-395.

[Mi] J. Milnor. Cubic polynomial maps with periodic critical orbit. I. In Complex dynamics, pages 333-411. A K Peters, Wellesley, MA, 2009.

Department of Mathematics, Statistics, and Computer Science, University of IlliNOIS AT Chicago.

E-mail address: demarco@math.uic.edu 